\title{
Effective use of latent semantic indexing and computational linguistics in biological and biomedical applications
}

\author{
Hongyu Chen ${ }^{1}$, Bronwen Martin'2, Caitlin M. Daimon² and Stuart Maudsley ${ }^{1 *}$ \\ ${ }^{1}$ Laboratory of Neuroscience, Receptor Pharmacology Unit, National Institute on Aging, National Institutes of Health, Baltimore, MD, USA \\ ${ }^{2}$ Laboratory of Clinical Investigation, Metabolism Unit, National Institute on Aging, National Institutes of Health, Baltimore, MD, USA
}

Edited by:

Firas H. Kobeissy, University of

Florida, USA

\section{Reviewed by:}

Bilal Fadlallah, University of Florida USA

Fadi A. Zaraket, American University of Beirut, Lebanon

Dan Xia, Harvard Medical School, USA

\section{${ }^{*}$ Correspondence:}

Stuart Maudsley, Laboratory of

Neuroscience, Receptor

Pharmacology Unit, National

Institute on Aging, National

Institutes of Health, 251 Bayview

Blvd., Baltimore, MD 21224, USA.

e-mail: maudsleyst@mail.nih.gov
Text mining is rapidly becoming an essential technique for the annotation and analysis of large biological data sets. Biomedical literature currently increases at a rate of several thousand papers per week, making automated information retrieval methods the only feasible method of managing this expanding corpus. With the increasing prevalence of open-access journals and constant growth of publicly-available repositories of biomedical literature, literature mining has become much more effective with respect to the extraction of biomedically-relevant data. In recent years, text mining of popular databases such as MEDLINE has evolved from basic term-searches to more sophisticated natural language processing techniques, indexing and retrieval methods, structural analysis and integration of literature with associated metadata. In this review, we will focus on Latent Semantic Indexing (LSI), a computational linguistics technique increasingly used for a variety of biological purposes. It is noted for its ability to consistently outperform benchmark Boolean text searches and co-occurrence models at information retrieval and its power to extract indirect relationships within a data set. LSI has been used successfully to formulate new hypotheses, generate novel connections from existing data, and validate empirical data.

Keywords: latent semantic indexing, data mining, computational linguistics, molecular interactions, drug discovery

\section{INTRODUCTION}

Over the past decade the ability for biomedical scientists to generate large-scale data sets has surpassed the processing capabilities of standard analytical tools. The high content and volume of large "omic" data sets make identification of key factors and the elucidation of cryptic data connections increasingly problematic. A sensible option for data analysis and information extraction is to preprocess the data to form distinct, functional groups. For many bioinformatic applications, this form of preprocessing is accomplished by clustering genes/proteins into pre-determined Gene Ontology (GO) term groups or canonical signaling pathways, e.g., KEGG (Kyoto Encyclopedia of Genes and Genomes) or BioCarta. However, this data organization relies upon the accuracy and fidelity of experimentally-driven human curation of these groups or pathways. These grouping functions may be artificially exclusive and also potentially outdated by subsequently-obtained experimental data. These systems, while providing an effective form of data analysis, are inherently rigid in their construction and therefore could be supplemented by using alternative strategies, e.g., Latent Semantic Indexing (LSI) or Latent Semantic Analysis (LSA). LSI is a commonly-used dimensionality-reduction technique used to compare similar "concepts/topics" among a collection of terms or documents. LSI is frequently employed in language processing to serve a variety of purposes, e.g., text categorization, indexing, essay grading, image auto-annotation, and automatic cross-language retrieval (Foltz and Dumais, 1992; Dumais et al., 1997; Deerwester et al., 1999; Sebastiani, 2002; Monay and Gatica-Perez, 2003). The utility of LSI stems from its ability to address multiple problems associated with other information retrieval methods: sparseness, noise, term independence, synonymy, and polysemy. Synonymy is defined as two terms conveying the same semantic meaning. Therefore, with a conventional Vector Space Model (VSM), two vectors could be similar even though their similarity lies in values from different dimensions (terms). Conversely, polysemy is defined as the same term having different meanings. Therefore, with a conventional VSM, two identical vectors can theoretically have different meanings. Term independence assumes that one term's presence does not affect any other terms currently in the document.

As the volume of textual information increases in the biomedical field, literature mining is becoming an effective approach to extract physiological meaning from such data sets. The interrogation of well curated bodies of accessible biomedical data, e.g., PubMed and the Gene Expression Omnibus, with LSI/LSA is likely to enhance our appreciation of complex, multifactorial disorders such as Alzheimer's disease. In this review, we will outline the mechanical structure of LSI-based approaches, demonstrate their ability to aid data extraction from mass data sets as well as discuss the relative benefits and drawbacks of such tools in the realm of biomedical data mining. 


\section{MECHANICS BEHIND LATENT SEMANTIC INDEXING}

LSI can be used on any corpus involving the use of conceptual identifiers, such as words of any language, identification numbers or letters, indices, morphemes, or any meaningful tokens. A matrix, M, is constructed from the corpus with each row representing the set of all terms, $\mathrm{T}$, and each column representing the set of all documents, D (Figure 1A). Each entry $\mathrm{a}_{\mathrm{ij}}$ in the matrix is positively defined by a weighting function if $T_{i} \in D_{j}$, and zero otherwise. Common weighting functions such as log-entropy, term frequency-inverse document frequency (tf-idf), and term frequency-normal (tf-normal) are used to underweigh common words and overweigh infrequent words that are likely to be more discriminatory identifiers of a document. The resulting matrix is referred to as the "term-document" matrix. An important trait of weighting functions such as tf-idf, tf-normal, and log-entropy is to map a discrete power law distribution, which is exemplified in the vast majority of natural language according to Zipf's law, into a continuous Gaussian function, a requirement for a later step, Singular Value Decomposition (SVD).

Next, SVD is performed on the term-document matrix M (Golub and Reinsch, 1970). Briefly, SVD factorizes the matrix into three matrices: $\Sigma$, a diagonal matrix with the square roots of the eigenvalues of $\mathrm{MM}^{\mathrm{T}}$ sorted in descending order; $\mathrm{U}$, a square matrix of dimensions $\mathrm{T} \times \mathrm{T}$ with each column representing the eigenvector of $\mathrm{MM}^{\mathrm{T}}$ that corresponds to each eigenvalue in $\Sigma$; and $\mathrm{V}^{\mathrm{T}}$, the transpose of a square matrix of dimensions $\mathrm{D} \times \mathrm{D}$ with each column representing the eigenvector of $\mathrm{M}^{\mathrm{T}} \mathrm{M}$ corresponding to each eigenvalue in $\Sigma$. For an arbitrary matrix $\mathrm{M}$, there exists at least one factorization into $U \Sigma V^{T}$ via SVD, where the singular value matrix is guaranteed to be unique. The original matrix $\mathrm{M}$ can be reconstructed by the equation $\mathrm{U} \Sigma \mathrm{V}^{\mathrm{T}}$.

The matrix $U$ is the term-concept matrix, with each entry representing a term's relation with a concept. Similarly, $\mathrm{V}^{\mathrm{T}}$

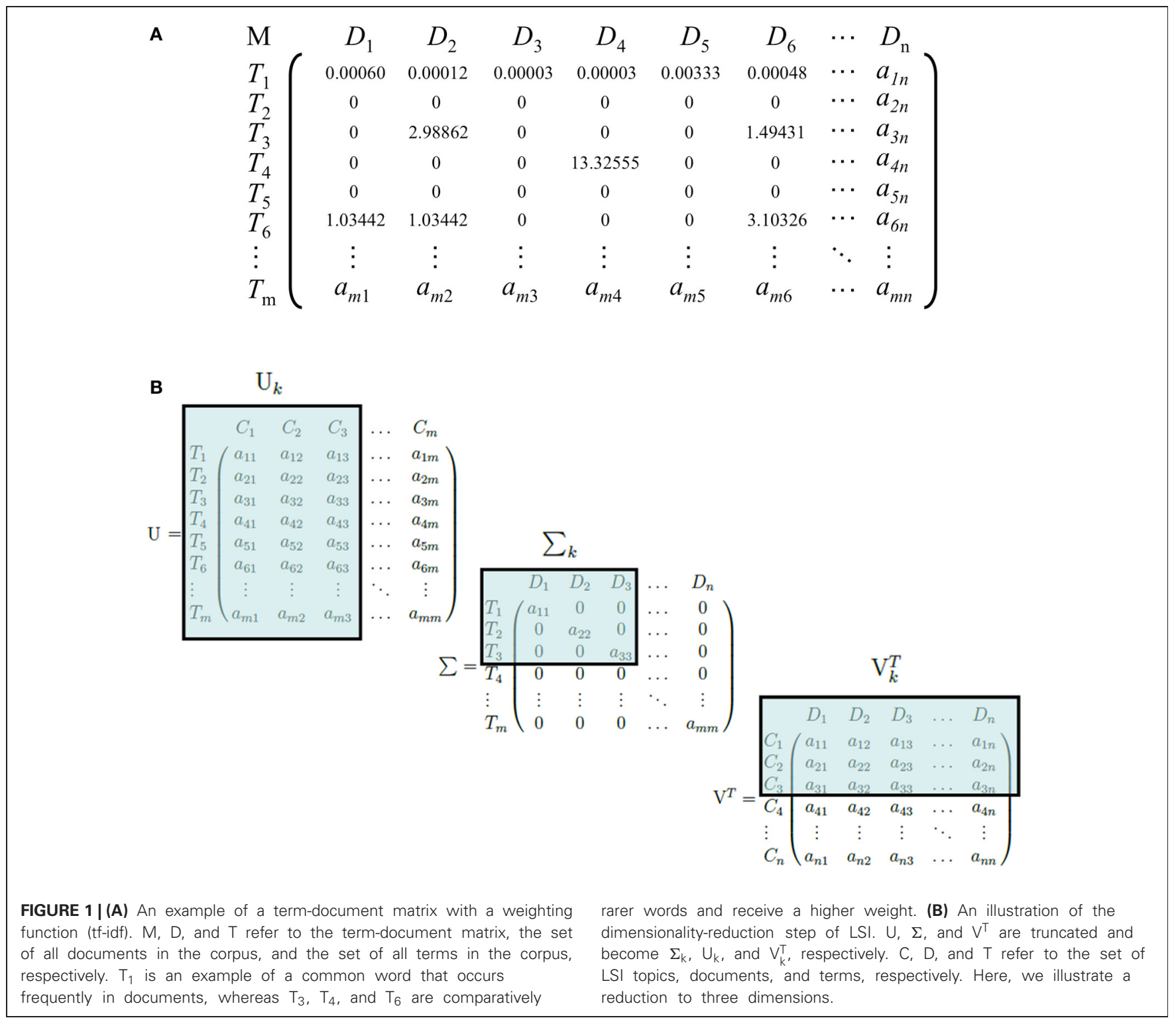


is the concept-document matrix, with each entry representing a document's relation with a concept. LSI then performs the dimensionality-reduction step by truncating each matrix. The top $k$ singular values are taken from $\Sigma$, because they capture the most variance from the original matrix, and the first $k$ columns and rows are taken from $U$ and $\mathrm{V}^{\mathrm{T}}$, respectively. The resulting matrices $\Sigma_{\mathrm{k}}, \mathrm{U}_{\mathrm{k}}$, and $\mathrm{V}_{\mathrm{k}}^{\mathrm{T}}$ capture the reduced-dimension representation of $\mathrm{M}$ (Figure 1B).

These matrices can then be used as a distance metric for both terms and documents. Any two documents can be compared by computing the cosine distance between their corresponding column vectors in $\mathrm{V}^{\mathrm{T}}$. Likewise, any two terms can be compared by computing the cosine distance between their corresponding row rectors in U. All user generated queries are treated as a separate document. However, SVD does not need to be repeated. Rather, since $\mathrm{M}=\mathrm{U} \Sigma \mathrm{V}^{\mathrm{T}}$ and therefore $\mathrm{V}=\mathrm{M}^{\mathrm{T}} \mathrm{U} \Sigma^{-1}$, one can index the user query $q$ by adding a new column to $M$ with the same weighting function, and right multiply the transpose by $U$ and $\Sigma^{-1}$ to attain the concept-document matrix. The query can then be compared to all existing documents using cosine distance (Berry et al., 1995).

Because of its use of various linear algebra techniques, LSI possesses many advantages over standard Boolean term searching and VSMs. First, LSI is used in conjunction with, and not instead of, common Boolean search weighting functions such as tf-idf. Term independence, an assumption of the standard VSM, which is false in some applications, is not assumed in LSI. Whereas 99\% of all entries in a typical term document matrix are zero, making sparseness a problem, most of the entries in the resulting LSI matrices are non-zero (Landauer et al., 1998). Noise is reduced during the dimensionality-reduction step, since the noise is assumed to be in the discarded columns and rows. LSI addresses synonymy by the fact that synonyms are commonly used in the same context and therefore LSI concepts are likely to reflect them. Polysemy is addressed, though debatably inadequately, by the noise reduction - as infrequent uses of a particular word may be discounted during the dimensionality-reduction step. LSI possesses advantages over other dimensionality reduction techniques such as covariance-based Principal Component Analysis (PCA). The latter performs an eigen-decomposition on the computed (square) covariance matrix, whereas LSI applies SVD directly on the (non-necessarily square) input matrix.

\section{ENHANCED DISTANCE METRIC OVER CONVENTIONAL MODELS}

Despite LSI's widespread usage in linguistics, it remains an underappreciated tool in biology. Often a comparison between two or more articles, genes or proteins is required for the analysis, clustering, categorization, and classification of such entities. The distance metric used for comparison is crucial for determining the quality of the algorithms that employ it. A high quality distance metric must tolerate sparseness, disregard noise and capture the intrinsic and extrinsic links between two entities. As a result, LSI can be used as an effective distance metric, and has been shown to outperform co-occurrence models and simple VSMs (Deerwester et al., 1999; Homayouni et al., 2005; Chagoyen et al., 2006; Klie et al., 2007; Ha et al., 2011; Roy et al., 2011; Xu et al., 2011).
LSI's enhanced distance metric stems from its robustness against noise, synonymy and polysemy due to reduced dimensionality.

A basic application of this distance metric is the measure of similarities among clinical documents. As previously mentioned, LSI is not dependent upon specific languages or grammars. Ha et al. applied LSI to a corpus of Korean discharge summaries and newspaper articles and noted that LSA-measured document similarities correlated with co-occurrence and was effective at measuring both Korean lexical morpheme-to-morpheme and document-to-document similarities (Ha et al., 2011). Using LSI's freedom from the necessity of grammatically-correct English language, biologists have frequently employed "gene documents" to a concatenation of all MEDLINE abstracts associated with a specific gene. "Gene documents" allow biologists to measure the similarity between two genes by mining the biomedical literature associated with each gene. LSI can be applied to these documents and all pairwise distance metrics among genes used for a variety of purposes, including agglomerative hierarchical clustering, determining the "cohesion" of a gene list and identifying transcription factor candidates (Homayouni et al., 2005; Roy et al., 2011; Xu et al., 2011). These tasks' precision and recall were evaluated on a "gold standard" set and deemed to outperform that obtained by benchmark co-occurrence methods. Similar results have been obtained for proteins (Chagoyen et al., 2006; Klie et al., 2007).

\section{LATENT LINKS FOR LITERATURE-BASED BIOMEDICAL DISCOVERY}

Literature-based discovery describes the problem of extracting previously unknown connections in two disjoint sets of scientific literature through the use of an intermediate set (Swanson, 1987, 1989, 1990). LSI's decreased dependence on direct term matches allows for the extraction of hidden relationships among concepts. For example, a hidden link can occur between the concepts denoted by term $\mathrm{A}$ and term $\mathrm{C}$ because of their respective co-occurrence with term $\mathrm{B}$, even though they do not co-occur themselves (Figure 2). This relationship, on the term level at least, is the core principle of Swanson discovery. Therefore LSI has been shown to be a powerful tool in identifying potential discoveries from the scientific literature without de facto empirical scientific demonstration of a direct linkage (Gordon and Dumais, 1998).

Kim et al. attempted to retrieve unrecognized gene relationships by using LSI along with Non-Negative Matrix Factorization (NMF), another matrix factorization method (Kim et al., 2007). Gene retrieval was evaluated on manually created test sets based on precision and recall, showing that LSI- and NMF-based methods vastly outperformed cooccurrence methods. Similarly, Roy et al. demonstrated LSI's ability to identify implicit links between transcription factors derived from a set of differentially expressed genes (Roy et al., 2011). ComputableGenomix's web-based semantic search engine, GeneIndexer, uses LSI on MEDLINE abstracts to identify known and unknown gene relationships. Typically, strongly correlated factors demonstrate cosine similarities in the document matrices near $0.4-0.7$ while implicit associations are only considered valid for further investigation with a cosine score 


$$
\text { Document }_{1}\left(\begin{array}{c}
\cdots \\
\text { term }_{a} \\
\operatorname{term}_{a} \\
\cdots \\
\cdots
\end{array}\right) \leftrightarrow \text { Document }_{2}\left(\begin{array}{c}
\cdots \\
\text { term }_{a} \\
\cdots \\
\text { term }_{b} \\
\cdots
\end{array}\right) \leftrightarrow \text { Document }_{3}\left(\begin{array}{c}
\cdots \\
\text { term }_{c} \\
\text { term }_{c} \\
\text { term }_{b} \\
\cdots
\end{array}\right)
$$

FIGURE 2 | An illustration of a "latent" link between term $_{\boldsymbol{a}}$ and term $_{\boldsymbol{c}}$ because of their respective co-occurrence with term $\boldsymbol{b}$. The principle of Swanson discovery is analogous to this-we have two currently disjointed sets of literature A and C and bridge the gap by introducing an intermediate literature set B.

of $>0.1$ (Homayouni et al., 2005; Roy et al., 2011). Using this discovery type of dataset interrogation biologists have been able to identify promising yet previously unknown links between genes and user-defined input words (Lee et al., 2007; Tijoe et al., 2008; Chadwick et al., 2011a).

\section{VALIDATION OF OBSERVATIONS AGAINST CURRENT LITERATURE}

LSI-based tools allow for the validation of experimental observations. Statistically significant differences amongst gene-keyword associations between experimental and control groups, using relevant keywords, can illustrate whether empirical observations are well-supported by the scientific literature. For example, Chadwick et al. used GeneIndexer to demonstrate that a much larger proportion of significant gene-keyword interactions existed in the Alzheimer's disease mouse model $(3 \times \mathrm{TgAD})$ group compared to the control group (Chadwick et al., 2010a). With experimentally relevant keywords like "Alzheimer's" and "oxidation," this finding coincided with experimental results, lending support to the experimental observations. Similar experiments have also used GeneIndexer as validation (Chadwick et al., 2010b, 2011b,c, 2012; Zhou et al., 2011). Using this LSI-based approach, accurate data "phenotypes" can be generated by using protagonistic and antagonistic gene-keyword combinations (Chadwick et al., 2010b). Therefore, a well-informed user can generate a gestalt appreciation of the potential functional inter-relationships of all of the genes/proteins in the original data set. Wei et al. used an LSI-based transcriptional factor identification method to validate the role of cRel as a regulator of interferon-simulated genes (Wei et al., 2008). One important aspect of literature mining is that the source of validation is constantly evolving. Literature-based discovery and LSI-based validation generate new scientific discoveries, which, when published in biomedical databases like PubMed, can be indexed again for future analysis.

\section{VISUALIZATION OF HIGH-DIMENSIONAL DATA}

LSI can be used to enhance visualization of data in two ways. First, it allows for extraction of information from unstructured or semi-structured corpora. LSI, in conjunction with other natural language processing techniques, can be used to interpret key concepts from a corpus and project it back to the user in graphical form. Jahiruddin et al. implemented this concept by creating BioKEVis, a search interface that produces semantic nets for the visualization of biomedical knowledge from PubMed (Jahiruddin et al., 2010). Second, LSI's ability to reduce dimensionality allows for a better visualization of high-dimensionality points that exceed the realm of physical space. For example, LSI can be used to reduce the number of dimensions in vector space to one, two, or three so that each point is graphable in threedimensional space (Kim et al., 2007). A major disadvantage to this method is that three dimensions is typically not an optimal value for $k$, so information loss will be significant. To maintain performance, dimensionality-reduction to an optimal $k$ can be performed to reduce noise, and then various high-dimensionality visualization techniques can be used to visualize the resulting, lower-dimensionality data (Swayne et al., 1992, 1998). With more accessible visualization of data, users can form their own interpretations of the data in addition to what has been presented by algorithmic analysis.

\section{LIMITATIONS OF LSI-BASED ANALYSES}

Though undoubtedly a useful tool, LSI does possess some disadvantages. The most obvious disadvantage is the selection of $k$, or the number of vectors in $\mathrm{U}$ and $\mathrm{V}^{\mathrm{T}}$ to keep. A high $k$ value may seem advantageous because one compares all documents across more concepts, but can be detrimental due to added noise. Conversely, a low $k$ value suffers from the danger of discarding crucial, distinguishing concepts in the data. This problem can be ameliorated to a certain extent by optimizing the precision and recall of LSI retrieval with a priori knowledge (Dumais, 2004; Kim et al., 2007). Analysis of the variance captured by the current dimensions, similar to that of PCA, is another method frequently employed (Cangelosi and Goriely, 2007). Overall, anywhere between 300 and 500 is appropriate for large corpora of millions of documents (Bradford, 2008). Another limitation of LSI is that it is computationally intensive. Calculating the SVD of a matrix $\mathrm{M}$ via reduction to a bidiagonal matrix has a computational complexity of $\mathrm{O}[m \times n \times \min (m, n)]$, where $m$ and $n$ are the number of rows and columns in M, respectively. For large term-document matrices, such computation is unfeasible. However, since only the reduce-rank matrix of the SVD of $M$ is used for LSI, one can perform "rank-reduced" SVD on M, yielding a computational complexity of $\mathrm{O}(m \times n \times k)$, which is more scalable (Jahiruddin et al., 2010). In addition, along with high $k$ values and inherent computational complexities, the future application of LSI to biomedical data may be hampered by the ever-increasing need for expanded data storage space. Finally, LSI 
uses the bag-of-words model when converting a corpus into the term-document matrix. The ordering of words in a document is completely disregarded, even though it is undoubtedly important. Despite this, there have been efforts to incorporate grammatical relations, sentence structure, and parts-of-speech tagging into LSI for biological fields (Klein and Manning, 2003; Brand, 2005; Settles, 2005).

\section{INTEGRATION OF LSI WITH CLASSICAL INFORMATICS}

With LSI-based information retrieval it is now possible to detect undiscovered molecular interactions. Even though standard data clustering/enrichment processes can only aid the interpretation of existing data, we cannot consider these approaches redundant. "Combinatorial informatics" comprises a synergistic combination of both LSI with standardized bioinformatic workflows. We have recently developed such a workflow to facilitate the discovery of biomolecular "keystone" factors (Chadwick et al., 2012). Mathematical modeling of "real-world" networks, has demonstrated that complex systems are not connected in an equitable and homogenous manner. Network connections can occur within small, tightly-connected "small-world networks" or between different "small-world networks" (Watts and Strogatz, 1998). From a biological standpoint, these "smallworlds" are analogous to biological processes such as kinase signaling cascades, while components of endocrine or neuronal axes could represent the constellations of these groups of small-world networks. Within global networks of genes/proteins, there are likely to exist specific genes/proteins that form the most important bridges between multiple "small-world" networks. Such genes/proteins within a functional network are often described as keystones. Keystones enhance rapid connectivity between disparate parts of a network and, as such, can be considered as functional "short-cuts" within the system (Watts and Strogatz, 1998). It has been shown that that even in networks commensurate with the biological scale (containing thousands to millions of nodes), surprisingly few (5-10) "short-cuts" are required to facilitate rapid information transfer across large systems (Watts and Strogatz, 1998). Classical KEGG/GO data set enrichment analysis can be transferred into LSI-based queries to assist in the discovery of keystone factors (genes/proteins) that possess a disproportionate ability to associate with the greatest number of the predicted KEGG/GO signaling paradigms.

\section{REFERENCES}

Ashburner, M., Ball, C. A., Blake, J. A., Botstein, D., Butler, H., Cherry, J. M., et al. (2000). Gene Ontology: tool for the unification of biology. The Gene Ontology Consortium. Nat. Genet. 25, 25-29.

Berry, M. W., Dumais, S. T., and O’Brien, G. W. (1995). Using linear algebra for intelligent information retrieval. SIAM Rev. 37, 575-595.

Blei, D. M., Ng, A. Y., and Jordan, M. I. (2003). Latent dirichlet allocation. J. Mach. Learn. Res. 3, 993-1022.

\section{CONCLUSION}

LSI has been successfully employed in a variety of biological contexts from the clustering of gene sets to the visualization of high-dimensionality data. Its ability to alleviate the effects of sparseness and noise, common traits of high-throughput "omics" data, makes textual analysis possible on data sets where standard term searching produces inadequate results. LSI is independent of the constraints of specific languages or grammars, thereby allowing researchers to employ gene documents, protein/experiment documents, and even noun-phrase documents to address the problem at hand. LSA, in the field of linguistics, has seen a wide variety of suggested improvements over the years. Probabilistic variants such as Probabilistic LSA and Latent Dirichlet Allocation have been suggested for their addition of a more accurate probabilistic model with respect to understanding of semantic concepts (Hofmann, 1999; Blei et al., 2003). Additional variants, including Hierarchical Dirichlet Processes and Random Projections, are interesting alternatives to LSI, that accomplish the same goals of dimensionality-reduction and topic modeling (Gionis et al., 1999; Teh et al., 2006). These methods, while not better or worse are certainly viable alternative candidates for biological data mining that should be evaluated alongside LSI. There seems however to be an unfortunate "lag" between developments in LSI and its integration with biomedically-related fields. For instance, PubMed was initially released in 1996, 6 years after the development of LSI. However, it was not until 2009 that PubMed released a searching algorithm not dependent upon outdated Boolean term searches. The most recent, state-of-the-art developments in computational linguistics and LSI/LSA may however require years, or even decades, to be accepted and used widely in the biological community. Nevertheless, with a conscious effort to improve data quality for literature mining with the use of standardized terms (MeSH, KEGG, GO), text mining is becoming increasingly viable and popular (Ashburner et al., 2000; Coletti and Bleich, 2001). With a realization of the importance of inter-disciplinary analysis and increased collaboration between biologists and computational linguists, there is the exciting possibility of rapid advancement in the field of literature mining as an important bioinformatics technique.

\section{ACKNOWLEDGMENTS}

This research was supported by the Intramural Research Program of the National Institute on Aging, National Institutes of Health.

application to cDNA microarray data. Biol. Direct 2, 2.

Chadwick, W., Brenneman, R., Martin, B., and Maudsley, S. (2010a). Complex and multidimensional lipid raft alterations in a murine model of Alzheimer's disease. Int. J. Alzheimers Dis. 2010:604792. doi: 10.4061/2010/ 604792

Chadwick, W., Zhou, Y., Park, S. S., Wang, L., Mitchell, N., Stone, M. D., et al. (2010b). Minimal peroxide exposure of neuronal cells induces multifaceted adaptive responses.
PLoS ONE 5:e14352. doi: 10.1371/ journal.pone.0014352

Chadwick, W., Boyle, J. P., Zhou, Y., Wang, L., Park, S. S., Martin, B. et al. (2011a). Multiple oxygen tension environments reveal diverse patterns of transcriptional regulation in primary astrocytes. PLoS ONE 6:e21638. doi: 10.1371/journal.pone.0021638

Chadwick, W., Keselman, A., Park, S. S., Zhou, Y., Wang, L., Brenneman, R., et al. (2011b). Repetitive peroxide exposure reveals pleiotropic mitogen-activated protein kinase 
signaling mechanisms. J. Signal Transduct. 2011:636951. doi: 10.1155/2011/636951

Chadwick, W., Mitchell, N., Caroll, J., Zhou, Y., Park, S. S., Wang, L., et al. (2011c). Amitriptyline-mediated cognitive enhancement in aged 3xTg Alzheimer's disease mice is associated with neurogenesis and neurotrophic activity. PLOS ONE 6:e21660. doi: 10.1371/journal. pone. 0021660

Chadwick, W., Martin, B., Chapter, M. C., Park, S. S., Wang, L., Daimon, C. M., et al. (2012). GIT2 acts as a potential keystone protein in functional hypothalamic networks associated with age-related phenotypic changes in rats. PLoS ONE 7:e36975. doi: 10.1371/journal.pone.0036975

Chagoyen, M., Carmona-Saez, P., Gil, C., Carazo, J. M., and PascualMontano, A. (2006). A literaturebased similarity metric for biological processes. BMC Bioinformatics 7:363. doi: 10.1186/1471-2105-7363

Coletti, M. H., and Bleich, H. L. (2001). Medical subject headings used to search the biomedical literature. J. Am. Med. Inform. Assoc. 8, 317-323.

Deerwester, S., Dumais, S. T., Furnas, G. W., Landauer, T. W., and Harshman, R. (1999). Indexing by latent semantic analysis. J. Am. Soc. Info. Sci. 41, 381-407.

Dumais, S. (2004). Latent semantic analysis. Ann. Rev. info. Sci. Tech. 38, 189-230.

Dumais, S. T., Landauer, T. K., and Littman, M. L. (1997). Automatic cross-linguistic information retrieval using latent semantic indexing. AAAI Tech. Rep. 1, 18-24.

Foltz, P. W., and Dumais, S. T. (1992). Personalized information delivery: an analysis of information filtering methods. Commun. ACM 34, 51-60.

Gionis, A., Indyk, P., and Motwani, R. (1999). "Similarity search in high dimensions via hashing," in Proceeding VLDB '99 Proceedings of the 25th International Conference on Very Large Data Bases (Burlington, MA: Morgan Kaufmann), 518-529.
Golub, G. H., and Reinsch, C. (1970). Singular value decomposition and least squares solutions. Numerische Mathematik 14, 403-420.

Gordon, M., and Dumais, S. (1998). Using latent semantic indexing for literature based discovery. J. Am. Soc. Info. Sci. 49, 674-685.

Ha, C., Yoo, S., and Choi, J. (2011). Evaluation of co-occurring terms in clinical documents using latent semantic indexing. Healthc. Inform. Res. 17, 24-28.

Hofmann, T. (1999). Probabilistic latent semantic analysis. Proc. ACM 22, 50-57.

Homayouni, R., Heinrich, K., Wei, L., and Berry, M. W. (2005). Gene clustering by latent semantic indexing of MEDLINE abstracts. Bioinformatics 21, 104-115.

Jahiruddin, M., Abulaish, M., and Dey, L. (2010). A concept-drive biomedical knowledge extraction of visualization framework for conceptualization of text corpora. J. Biomed. Inform. 43, 1020-1035.

Kim, H., Park, H., and Drake, B. (2007). Extracting unrecognized gene relationships from the biomedical literature via matrix factorizations. BMC Bioinformatics 2007, 8:S6. doi: 10.1186/1471-2105-8-S9-S6

Klein, D., and Manning, C. D. (2003). "Accurate unlexicalized parsing," in Proceedings of the 41st Meeting of the Association for Computational Linguistics (Stroudsburg, PA: ACL), 423-430.

Klie, S., Martens, L., Vizcaino, J. A., Cote, R., Jones, P., Apweiler, R., et al. (2007). Analyzing large-scale proteomics projects with latent semantic indexing. J. Proteome Res. 7, 182-191.

Landauer, T. K., Foltz, P. W., and Laham, D. (1998). Introduction to latent semantic analysis. Discourse Process. 25, 259-284.

Lee, S., Xu, L., Shin, Y., Gardner, L., Hartzes, A., Dohan, F. C., et al. (2007). A potential link between autoimmunity and neurodegeneration in immune-mediated neurological disease. J. Neuroimmunol. 235, 58-69.
Monay, F., and Gatica-Perez, D. (2003). "On image auto-annotation with latent space models," in Proceedings of the 11th ACM International Conference (New York, NY: ACM), 275-278.

Roy, S., Heinrich, K., Phan, V., Berry, M. W., and Homayouni, R. (2011). Latent semantic indexing of PubMed abstracts for identification of transcription factor candidates from microarray derived gene sets. BMC Bioinformatics 12:S19. doi: 10.1186/1471-2105-12S10-S19

Sebastiani, F. (2002). Machine learning in automated text categorization. ACM Comput. Surv. 24, 1-47.

Settles, B. (2005). ABNER: an open source tool for automatically tagging genes, proteins, and other entity names in text. Bioinformatics 21, 3191-3192.

Swanson, D. R. (1987). Two medical literatures that are logically but not bibliographically connected. J. Am. Soc. Inform. Sci. 38, 228-233.

Swanson, D. R. (1989). Online search for logically related noninteractive medical literatures: a systematic trial and error strategy. J. Am. Soc. Inf. Sci. 40, 356-358.

Swanson, D. R. (1990). Medical literature as a potential source of new knowledge. Bull. Med. Libr. Assoc. 78, 29-37.

Swayne, D. F., Cook, D. H., Buja, A., and Gobi, X. (1992). "Interactive dynamic graphics in the X Window system with a link to S," in American Statistical Association 1991 Proceedings of the Section on Statistical Graphics (Alexandria, VA: The Association), 1-8.

Swayne, D. F., Cook, D. H., Buja, A., and Gobi, X. (1998). Interactive dynamic data visualization in the $\mathrm{X}$ Window System. J. Comput. Graph. Stat. 7, 113-130.

Teh, Y. W., Jordan, M. I., Beal, M. J., and Blei, D. M. (2006). Hierarchical dirichlet processes. J. Am. Stat. Assoc. 101, 1566-1581.

Tijoe, E., Berry, M. W., and Homayouni, R. (2008). Using a literature based NMF model for discovering gene functional relationships. BMC Bioinformatics
9:P1. doi: 10.1186/1471-2105-9S7-P1

Watts, D. J., and Strogatz, S. H. (1998). Collective dynamics of 'small-world' networks. Nature 393, 440-442.

Wei, L., Fan, M., Xu, L., Heinrich, K., Berry, M. W., Homayouni, R., et al. (2008). Bioinformatic analysis reveals cRel as a regulator of a subset of interferon-simulated genes. J. Interferon Cytokine Res. 28, 541-551.

Xu, L., Furlotte, N., Lin, Y., Heinrich, K., Berry, M. W., George, E. O., et al. (2011). Functional cohesion of gene sets determined by latent semantic indexing of PubMed abstracts. PLoS ONE 6:e18851. doi: 10.1371/ journal.pone.0018851

Zhou, Y., Yi, T., Park, S. S., Shen, R. F., Wu, W. W., Martin, B., et al. (2011). Rapid and enhanced proteolytic digestion using electricfield-oriented enzyme reactor. J. Proteomics 74, 1030-1035.

Conflict of Interest Statement: The authors declare that the research was conducted in the absence of any commercial or financial relationships that could be construed as a potential conflict of interest.

Received: 12 October 2012; paper pending published: 29 October 2012; accepted: 09 January 2013; published online: 30 January 2013.

Citation: Chen H, Martin B, Daimon CM and Maudsley S (2013) Effective use of latent semantic indexing and computational linguistics in biological and biomedical applications. Front. Physio. 4:8. doi: 10.3389/fphys.2013.00008

This article was submitted to Frontiers in Systems Biology, a specialty of Frontiers in Physiology.

Copyright () 2013 Chen, Martin, Daimon and Maudsley. This is an open-access article distributed under the terms of the Creative Commons Attribution License, which permits use, distribution and reproduction in other forums, provided the original authors and source are credited and subject to any copyright notices concerning any third-party graphics etc. 